\title{
TRANSCENDENCE OF FACTORIAL SERIES WITH PERIODIC COEFFICENTS
}

\author{
VERNE E. DIETRICH AND ARTHUR ROSENTHAL
}

It is well known that every real number $\alpha$ can be represented by a factorial series

$$
\alpha=\frac{a_{1}}{1 !}+\frac{a_{2}}{2 !}+\frac{a_{3}}{3 !}+\cdots+\frac{a_{n}}{n !}+\cdots,
$$

where the $a_{n}(n=1,2, \cdots)$ are integers and, moreover, $0 \leqq a_{n}<n$ (for $n=2,3, \cdots)$. This representation is unique for the irrational numbers $\alpha$, while every rational $\alpha$ can be represented either with almost all $^{1} a_{n}=0$ or with almost all $a_{n}=n-1$.

The representation (1) was discussed and the aforesaid properties were proved by M. Stéphanos [1]. ${ }^{2}$ But an even more general type of series had already been studied by G. Cantor [2] (not known to M. Stéphanos). These series have later been called "Cantor series" (cf. [3]).

In this note we consider the case in which the factorial series (1) has periodic coefficients $a_{n}$ and we prove the following theorem:

THEOREM 1. Every number $\alpha$ represented by a factorial series (1) with periodic coefficients is transcendental (except for the trivial case where almost all $a_{n}$ are zero).

The above condition $0 \leqq a_{n}<n$ (for $n=2,3, \cdots$ ) is not used at all in the following proof. Moreover, the coefficients $a_{n}$ need not necessarily be integers; the $a_{n}$ can be any algebraic numbers. Then Theorem 1 and its proof still hold.

We generalize Theorem 1 further:

THEOREM 2. If the power series ${ }^{3}$

$$
\phi(z)=\sum_{n=1}^{\infty} \frac{a_{n}}{n !} z^{n}
$$

has algebraic coefficients $a_{n}$ (not almost all of them being zero) which form a periodic sequence, then $\phi(z)$ is a transcendental number for every

Presented to the Society, November 26, 1948; received by the editors August 12, 1948.

1 The expression "almost all" is used in the sense of "all but a finite number."

${ }^{2}$ Numbers in brackets refer to the bibliography at the end.

3 Under the conditions of Theorem 2, $\phi(z)$ is an entire function. 
algebraic $z(\neq 0)$.

For $z=1$ Theorem 2 furnishes Theorem 1. Hence we prove Theorem 2 .

The general case in which the period starts with $a_{s}(s \geqq 1)$ can immediately be reduced to the case in which the period begins with $a_{1}$. One has only to subtract and add $s-1$ terms, that is, algebraic numbers. Therefore it suffices to assume that the period starts at $a_{1}$.

Let $m$ be the "length" of the period, that is, the number of coefficients $a_{n}$ belonging to the period, and let $\omega$ be a primitive $m$ th root of unity. Then

$$
\begin{aligned}
e^{\omega k \cdot z}=1 & +\frac{\omega^{k} \cdot z}{1 !}+\frac{\omega^{k 2} \cdot z^{2}}{2 !}+\cdots \\
& +\frac{\omega^{k(m-1)} \cdot z^{(m-1)}}{(m-1) !}+\frac{\omega^{k m} \cdot z^{m}}{m !}+\cdots
\end{aligned}
$$

Thus, since the sum of the $r$ th powers of all the $m$ th roots of unity is zero if $r$ is not divisible by $m$, we obtain:

$$
\begin{aligned}
\sum_{k=1}^{m} e^{\omega k \cdot z}=m & +0+\cdots+0+m \frac{z^{m}}{m !} \\
& +0+\cdots+0+m \frac{z^{2 m}}{(2 m) !}+\cdots
\end{aligned}
$$

and hence

$$
\begin{aligned}
\frac{a_{m}}{m} \sum_{k=1}^{m} e^{\omega k \cdot z}=a_{m} & +0+\cdots+0+a_{m} \frac{z^{m}}{m !} \\
& +0+\cdots+0+a_{m} \frac{z^{2 m}}{(2 m) !}+0+\cdots
\end{aligned}
$$

Similarly for $r=1,2, \cdots, m-1$ we have

$$
\begin{aligned}
\frac{a_{m-r}}{m} \sum_{k=1}^{m} \omega^{k r} e^{\omega k \cdot z}=0+ & \cdots+0+a_{m-r} \frac{z^{m-r}}{(m-r) !} \\
& +0+\cdots+0+a_{m-r} \frac{z^{2 m-r}}{(2 m-r) !}+0+\cdots .
\end{aligned}
$$

By summing over $r=0,1,2, \cdots, m-1$, we obtain

$$
\sum_{k=1}^{m} e^{\omega k \cdot z}\left(\frac{1}{m} \sum_{r=0}^{m-1} a_{m-r} \omega^{k r}\right)=\phi(z)+a_{m}
$$


or, if we $\operatorname{set}(1 / m) \sum_{r=0}^{m-1} a_{m-r} \omega^{k r}=A_{k}(k=1,2, \cdots, m)$, we have

$$
\sum_{k=1}^{m} A_{k} e^{\omega k \cdot z}-\left[\phi(z)+a_{m}\right] \cdot e^{0}=0 .
$$

It is impossible that all coefficients $A_{k}(k=1,2, \cdots, m)$ vanish; for then the equation of $(m-1)$ st degree

$$
\sum_{r=0}^{m-1} a_{m-r} z^{r}=0
$$

would have $m$ different roots $\omega^{k}(k=1,2, \cdots, m)$, while the case that all $a_{n}=0(n=1,2, \cdots, m)$ has been excluded. But now, since for an algebraic $z \neq 0$ the numbers $\omega^{k} \cdot z(k=1,2, \cdots, m)$ are algebraic, different, and nonzero and since the $A_{k}(k=1,2, \cdots, m)$ are also algebraic numbers and at least one of them is not zero, it follows from (3) by Lindemann's general theorem $([4,5]$, cf. also [6]) that $\phi(z)$ $+a_{m}$ and hence also $\phi(z)$ is a transcendental number for every algebraic $z \neq 0$.

\section{BIBLIOGRAPHY}

1. M. Stéphanos, Sur une propriêté remarquable des nombres incommensurables, Bull. Soc. Math. France vol. 7 (1879) pp. 81-83.

2. G. Cantor, Ueber die einfachen Zahlensysteme, Zeitschrift für Mathematik und Physik vol. 14 (1869) pp. 121-128.

3. O. Perron, Irrationalzahlen, Göschens Lehrbücherei, vol. 1, Berlin, 1st ed., 1921, 2d ed., 1939, 3d ed., 1947, \$33.

4. F. Lindemann, Ueber die Zahl $\pi$, Math. Ann. vol. 20 (1882) pp. 213-225.

5. - Ueber die Ludolph'sche Zahl, Sitzungsberichte der Akademie der Wiss. Berlin (1882) pp. 679-682.

6. K. Weierstrass, Zu Lindemann's Abhandlung: "Ueber die Ludolph'sche Zahl", Sitzungsberichte der Akademie der Wissenschaften, Berlin (1885) pp. 1067-1086.

Purdue University 